\title{
Simple model with facilitated dynamics for granular compaction
}

\author{
J. Javier Brey and A. Prados \\ Departamento de Física Teórica, Facultad de Física, Universidad de Sevilla, Apartado de Correos 1065, E-41080 Sevilla, Spain \\ B. Sánchez-Rey \\ Departamento de Física Aplicada, E. U. Politécnica, Universidad de Sevilla, Virgen de África 7, E-41011 Sevilla, Spain
}

(Received 24 November 1998; revised manuscript received 23 March 1999)

\begin{abstract}
A simple lattice model is used to study compaction in granular media. As in real experiments, we consider a series of taps separated by large enough waiting times. The relaxation of the density exhibits the characteristic inverse logarithmic law. Moreover, we have been able to identify analytically the relevant time scale, leading to a relaxation law independent of the specific values of the parameters. Also, an expression for the asymptotic density reached in the compaction process has been derived. The theoretical predictions agree fairly well with the results from the Monte Carlo simulation. [S1063-651X(99)17810-3]
\end{abstract}

PACS number(s): 81.05.Rm, 05.50.+q, 81.20.Ev

\section{INTRODUCTION}

One of the characteristic complex behaviors exhibited by granular materials is compaction [1-4]. It can be roughly defined as the density relaxation of a loosely packed system of many grains under mechanical tapping or vibration. Granular compaction is important to many industrial applications related with the production and manipulation of a wide variety of systems composed by many macroscopic particles or grains [2]. In the last few years, a series of experiments have been carried out trying to identify the physical principles underlying granular compaction $[2,3,5]$. Starting from a loosely packed initial configuration, systems of monodisperse glass beads were tapped vertically. The waiting time between successive taps was large enough to allow the system to relax, so that the beads were at rest before the next tap started. The time evolution of the density towards a steady state has been analyzed, and it has been shown that it can be accurately described by an inverse logarithmic law with four adjustable parameters, whose values depend only on the tapping strength measured by the peak acceleration of a tap. The logarithmic relaxation has been found in many different models [6-8] suggesting that such a behavior is quite general [9]. Although several mechanisms have been proposed to explain the behavior observed in the experiments, a fully satisfactory theory is still lacking.

Here we consider a one-dimensional model simple enough as to allow some detailed calculations. One of our aims was to try to identify the relevant time scale over which the relaxation (compaction) of the system takes place. This is the first step in the search of general laws governing the physics of densification. A main difficulty in studying compaction is that there are two different series of elementary processes involved in the experiment. The system is submitted to taps or pulses separated by time intervals for which the system is allowed to relax freely. The initial state for each tap is the final state from the previous relaxation. Both processes, tapping and free evolution, must be considered in detail, and they are rather different from a physical point of view. For instance, while the duration of the pulse is clearly a relevant parameter of the problem, the free relaxation is assumed to last by definition until the system gets trapped and it is at rest. Quite interestingly, the experiments have shown that it is useful to measure time by the number of pulses applied to the system. Another central question is how much settling will occur for a given vibration intensity, and also if the stationary value of the density depends on the initial configuration. How these facts appear in our model and which is the role played by the duration and amplitude of the pulses are points we will address here.

The plan of the paper is as follows. In the next section the model will be presented. It consists of a lattice whose sites can be occupied by particles. The dynamics is formulated by means of a master equation and it is facilitated, in the sense that the rates of adsorption and desorption of a particle are proportional to the number of particles in the nearest neighbor sites. The model can be exactly solved in the no desorption limit, which corresponds to the very low temperature limit. The solution is obtained in Sec. III and describes the evolution of the system without external perturbation. Therefore, it will be used to study the relaxation of the system towards a metastable state between pulses. In spite of the simplicity of the system, the general solution for arbitrary strength of the external energy source is rather complicated. We have considered the limit of short duration of the taps, not only because of mathematical convenience, but also because it seems to be the limit in which the time scales involved in the problem become well separated.

The sequence of taps and free relaxation processes, i.e., compaction is the subject of Sec. IV. An expression for the density after the $n+1$ tap in terms of the density and the probability distribution of two holes separated by a site after the previous tap is derived. Although this relation does not provide an explicit expression for the evolution of the density, it allows to identify the relevant time scale, which turns out to be proportional to the duration of a tap times a parameter measuring their strength. Curves describing the density evolution of systems starting from the same initial state but corresponding to different values of the parameters are shown to be the same when plotted as functions of the scaled time. Besides, the single scaled curve is very well fitted by the inverse logarithmic law known from real experiments. 
In the limit of many taps, the density reaches a steady value that is discussed in Sec. V. By using a pair approximation it is found that the steady density is proportional to the time relevant parameter mentioned above. This prediction agrees well with the numerical results from the simulations. Finally, the last section contains some final remarks and comments.

\section{DESCRIPTION OF THE MODEL}

We consider a one-dimensional lattice with $N$ sites. Each site can be either occupied by a particle or empty. A configuration of the system is specified, for instance, by giving an ordered sequence of $N$ particles and holes. Let us introduce a set of variables $m \equiv\left\{m_{i} ; i=1,2, \ldots, N\right\}$, such that $m_{i}$ vanishes if there is a particle at site $i$, while it takes the value 1 if there is a hole; i.e., the site $i$ is empty.

The dynamics of the system is defined as a Markov process and formulated by means of the master equation for the conditional probability $p_{1 / 1}\left(\mathbf{m}, t \mid \mathbf{m}^{\prime}, t^{\prime}\right)$ of finding the system in the configuration $m$ at time $t$, given it was in the configuration $m^{\prime}$ at time $t^{\prime}<t[10]$,

$$
\begin{aligned}
\frac{\partial}{\partial t} p_{1 / 1}\left(\mathbf{m}, t \mid \mathbf{m}^{\prime}, t^{\prime}\right)= & \sum_{i}\left[W_{i}\left(R_{i} \mathbf{m}\right) p_{1 / 1}\left(R_{i} \mathbf{m}, t \mid \mathbf{m}^{\prime}, t^{\prime}\right)\right. \\
& \left.-W_{i}(\mathbf{m}) p_{1 / 1}\left(\mathbf{m}, t \mid \mathbf{m}^{\prime}, t^{\prime}\right)\right],
\end{aligned}
$$

where $R_{i} \mathbf{m} \equiv\left\{m_{1}, \ldots, R_{i} m_{i}, \ldots, m_{N}\right\}$ with $R_{i} m_{i}=1-m_{i}$, i.e., $R_{i} \mathbf{m}$ is the configuration obtained from $\mathbf{m}$ by changing the state of hole or particle of site $i$. The above equation is to be solved with the initial condition

$$
p_{1 / 1}\left(\mathbf{m}, t^{\prime} \mid \mathbf{m}^{\prime}, t^{\prime}\right)=\delta_{\mathbf{m}, \mathbf{m}^{\prime}}=\prod_{i=1}^{N} \delta_{m_{i} m_{i}^{\prime}} .
$$

The one-time distribution

$$
p(\mathbf{m}, t)=\sum_{\mathbf{m}^{\prime}} p_{1 / 1}\left(\mathbf{m}, t \mid \mathbf{m}^{\prime}, 0\right) p\left(\mathbf{m}^{\prime}, 0\right)
$$

also obeys Eq. (1), although now the initial condition must be given in each specific situation.

The possible elementary processes occurring in the system are the adsorption of a particle on an empty site from a surrounding bulk and the desorption of a particle from the lattice to the bulk. Both processes are restricted in the following way. A particle can be adsorbed on or desorbed from a site only if at least one of its nearest neighbor sites is empty. More precisely, the probability rate for the events is proportional to the number of nearest neighbor holes. This condition tries to model naively the short-ranged geometrical constraints that make structural rearrangements difficult in a granular material. Thus the probability that an adsorption attempt be made on site $i$ in the infinitesimal time interval between $t$ and $t+d t$ is $k_{+} n_{i} d t$, where $n_{i}$ is the number of nearest neighbors holes of site $i$. Of course, a particle can be adsorbed only if the site is empty. In the same way, the probability per unit of time that a given particle try to leave the lattice is $k_{-} n_{i}$. Therefore, we assume that the transition rates are given by

$$
\begin{aligned}
& W_{i}\left(\mathbf{m}^{(i)}, m_{i}=1\right)=k_{+} \frac{m_{i-1}+m_{i+1}}{2}, \\
& W_{i}\left(\mathbf{m}^{(i)}, m_{i}=0\right)=k_{-} \frac{m_{i-1}+m_{i+1}}{2},
\end{aligned}
$$

with $m^{(i)}=\left\{m_{1}, \ldots, m_{i-1}, m_{i+1}, \ldots, m^{N}\right\}$, and we have used that the number of nearest neighbor holes is $n_{i}=m_{i-1}$ $+m_{i+1}$. The factor 2 in the denominators of Eq. (4) will simplify subsequent calculus. A similar kind of facilitated dynamics has been used previously in the context of Ising models [11]. Although we restrict ourselves here to the onedimensional case, the model can be formulated for arbitrary dimension. A possible physical interpretation of this facilitated dynamics in the context of granular media is to identify a hole with a "region" of the granular system that has lower than average packing fraction, and a particle with a region which has higher than average packing fraction. Then a low packing fraction region can facilitate a neighboring region to change its state, because the first region can respond to fluctuations of the latter region. On the other hand, a high packing fraction region would not be able to respond to such fluctuations in neighboring regions, and slowing down the dynamics of the system.

Let us introduce new constant parameters $\nu$ and $\epsilon$ by

$$
\nu=k_{-}+k_{+}, \quad \epsilon=\frac{k_{-}}{k_{-}+k_{+}} .
$$

The constant $\nu$ has the dimensions of a frequency and $\epsilon$ is a dimensionless parameter defined in the interval $0 \leqslant \epsilon \leqslant 1$. For $\epsilon=1$ no particle is adsorbed by the system, while for $\epsilon=0$ desorption processes do not occur. In terms of these parameters, Eqs. (4) can be written together as

$$
W_{i}(\mathbf{m})=\frac{\nu}{2}\left(m_{i-1}+m_{i+1}\right)\left[\epsilon+m_{i}(1-2 \epsilon)\right] .
$$

The ratio of the desorption transition rates to the adsorption ones is

$$
\frac{W_{i}\left(\mathbf{m}^{(i)}, m_{i}=0\right)}{W_{i}\left(\mathbf{m}^{(i)}, m_{i}=1\right)}=\frac{k_{-}}{k_{+}}=\frac{\epsilon}{1-\epsilon}=x,
$$

where the last equality defines the parameter $x$.

The stochastic process we have formulated has a steady one-time distribution of the form

$$
p_{s t}(\mathbf{m})=\frac{1}{(1+x)^{N}} \prod_{i=1}^{N} x^{m_{i}}
$$

and the density of holes (average number of holes divided by the total number of sites $N$ ) in the steady state is

$$
\left\langle m_{i}\right\rangle_{s t}=\sum_{\mathbf{m}} m_{i} p_{s t}(\mathbf{m})=\frac{x}{1+x}=\boldsymbol{\epsilon},
$$

and, consequently, the steady density of particles is $\rho_{s t}=1$ $-\epsilon$. As $x$ increases, the equilibrium density of particles decreases. 
The quantity $x(\epsilon)$ can be related with a temperature parameter $T$ by defining an energy $E(m)$ for the system. A possible choice is

$$
E(\mathbf{m})=e_{0} \sum_{i=1}^{N} m_{i}
$$

where $e_{0}$ is a constant fixing the energy scale. If now the distribution given by Eq. (8) is identified with the equilibrium canonical distribution, it is easily obtained that

$$
x=e^{-\beta e_{0}},
$$

with $\beta=\left(k_{B} T\right)^{-1}, k_{B}$ being the Boltzmann constant. Thus the limit $x \rightarrow \infty(\epsilon \rightarrow 1)$ is equivalent to $T \rightarrow 0^{-}$and the limit $x \rightarrow 0 \quad(\epsilon \rightarrow 0)$ to $T \rightarrow 0^{+}$. A purely random distribution of particles and holes corresponds formally to the equilibrium distribution for $\epsilon=1 / 2$ or $T \rightarrow \infty$. With the above definitions of energy and temperature, our system can be related to the family of kinetic Ising models introduced in Ref. [11] to study glassy relaxation. Nevertheless, it must be stressed that in the context of granular systems this temperature does not have the usual meaning, but it is related to the strength of the tapping process. So, we cannot expect a "thermodynamic", theory based on this concept of temperature to apply to compact granular systems [12].

The transition rates given in Eq. (7) define an irreducible Markov process for $\epsilon>0$, except for the state with all the sites occupied by particles that can not evolve. In the limit $N \rightarrow \infty$, the probability of this state is negligible, and all the solutions of the master equation relax to the steady distribution given by Eq. (8) [10]. The situation is different in the no desorption limit $\epsilon=0$. The density of particles cannot decrease, and all the states of the system having every hole surrounded by two particles are absorbent; no evolution is possible from them.

Our one-dimensional lattice model can be regarded as a very simple picture of an horizontal section of a real granular system, near the bottom of the container. Consider first the freely evolving case. In a real granular medium, particles cannot go up due to gravity. They can only go down, as long as there is enough empty space in their surroundings. Therefore, the packing fraction grows until the hard-core interaction prevents more movements of particles, and a mechanically stable configuration is found. This situation is naively resembled by the evolution of our model in the no desorption limit, $\epsilon=0$. Starting from a given configuration of particles and holes, the system evolves by means of adsorption processes, occurring on those sites having at least one nearest neighbor hole. This leads to a monotonic increase of the density until all the holes become isolated, i.e., surrounded by two particles.

Next, suppose a granular system submitted to vertical vibration. During the vibration, particles belonging to a low horizontal section can go up, making the local packing fraction decrease. The hard-core repulsion is also fundamental in the vibrated case, since particles always need enough free volume close to them in order to move. In our model, these pulses are introduced by allowing particles to be desorbed, but the dynamics is "facilitated." A particle can only be adsorbed or desorbed if at least one of its nearest neighbor sites is empty. This is done to mimic the short-ranged dynamical constraints in the real granular system. Of course, the relative magnitude $\epsilon$ of the desorption rate and the pulse duration $t_{0}$ are the parameters characterizing the process.

Then, tapping processes have been modeled in our lattice system in the following way. We started from a purely random configuration, i.e., the equilibrium configuration for $\epsilon$ $=1 / 2$. Then, the system was allowed to relax with $\epsilon=0$ until reaching a steady metastable configuration, characterized by all the holes being isolated, from which the system can not evolve any more. This is a convenient initial state for the compaction experiment and corresponds to the loosely packed conditions used in real laboratory experiments [2]. In this way the average initial density of particles in our tapping process has been $\rho \simeq 0.7$.

Pulses are modeled by suddenly increasing the value of $\epsilon$ to a value greater than zero. This is equivalent to increase the temperature of the system. The duration of each pulse was $t_{0} \ll 1$. Between pulses the system relaxes with no external excitation, i.e., with $\epsilon=0$. The waiting time between consecutive pulses was much larger than the relaxation time needed for the system to become trapped in a new metastable configuration. The density was measured just before starting a new pulse. The whole process was designed to mimic what is done in real experiments.

\section{EVOLUTION WITH CONSTANT TRANSITION RATES}

In this section we will study the evolution equations that determine the relaxation of the density. First, we will derive these equations for an arbitrary value of the parameter $\epsilon$ characterizing the relative probability of a desorption event. Secondly, we will analyze the free relaxation without desorption, i.e., in the limit $\epsilon=0$, and the effect of pulses separately, taking into account that the final state for one of the processes gives the initial condition for the other.

In the following we will restrict ourselves to homogeneous and isotropic states. This requires to consider appropriate initial and boundary conditions, and it is consistent with the qualitative picture depicted in the previous Section. It will be assumed that the limit $N \rightarrow \infty$ has been taken. Let us define probability distributions of groups of $r+1$ consecutive holes by

$$
D_{r}(t) \equiv\left\langle m_{i} m_{i+1} \cdots m_{i+r}\right\rangle_{t}=\sum_{m} m_{i} m_{i+1} \cdots m_{i+r} p(\mathbf{m}, t) .
$$

The homogeneity of the system implies that the above expression does not depend on the starting site $i$ considered. Evolution equations for the moments $D_{r}(t)$ are easily obtained from the master equation,

$$
\begin{gathered}
\frac{\partial}{\partial t} D_{0}(t)=\epsilon D_{0}(t)-D_{1}(t), \\
\frac{\partial}{\partial t} D_{1}(t)=\epsilon\left[D_{0}(t)+C_{0,0}(t)\right]-D_{1}(t)-D_{2}(t),
\end{gathered}
$$




$$
\begin{aligned}
\frac{\partial}{\partial t} D_{r}(t)= & -r D_{r}(t)-D_{r+1}(t)+\epsilon C_{0, r-1}(t) \\
& +\epsilon\left[D_{r-1}(t)+\sum_{j=1}^{r-1} C_{j-1, r-j-1}(t)\right] .
\end{aligned}
$$

for $r \geqslant 2$. Here we have introduced the probability distributions of two groups of holes separated by a site

$$
C_{r, s}(t)=\left\langle m_{i} m_{i+1} \cdots m_{i+r} m_{i+r+2} \cdots m_{i+r+s+2}\right\rangle_{t} .
$$

Besides, from now on we use the dimensionless time scale defined by $t^{*}=\nu t$, although the asterisk is omitted for the sake of simplicity. Again as a consequence of homogeneity, the functions $C_{r, s}(t)$ do not depend on the site $i$ taken as the origin to measure them. Moreover, isotropy implies the symmetry property $C_{r, s}(t)=C_{s, r}(t)$. In Eq. (13) it is seen that the time evolution of the density of holes $D_{0}(t)$ involves the nearest neighbor pair distribution of holes $D_{1}(t)$. When the equation (14) for this latter distribution is considered, the situation becomes more complex. Not only the three consecutive hole distribution $D_{2}(t)$ shows up, but also the second neighbor pair moment $C_{0,0}(t)$ appears.

On the other hand, the whole hierarchy of equations gets much simpler in the limit $\epsilon \rightarrow 0$. As discussed in Sec. II this is the no desorption limit and corresponds to $T \rightarrow 0^{+}$(very low temperatures). For $\epsilon=0$, Eqs. (13) $-(15)$ reduce to

$$
\frac{\partial}{\partial t} D_{r}^{(0)}(t)=-r D_{r}^{(0)}(t)-D_{r+1}^{(0)}(t)
$$

for all $r$. Hereafter, the superindex 0 indicates that a quantity is evaluated in a system evolving with $\epsilon=0$. The hierarchy (17) can be easily solved by using, for instance, the generating function method [10]. We introduce a generating function

$$
G^{(0)}(y, t)=\sum_{r=0}^{\infty} \frac{y^{r}}{r !} D_{r}^{(0)}(t) .
$$

From Eqs. (17) it is obtained that $G^{(0)}(y, t)$ obeys the equation

$$
\frac{\partial}{\partial t} G^{(0)}(y, t)+(y+1) \frac{\partial}{\partial y} G^{(0)}(y, t)=0,
$$

whose solution is

$$
G^{(0)}(y, t)=G_{0}\left[(y+1) e^{-t}-1\right],
$$

where $G_{0}(y)=G^{(0)}(y, 0)$ is the initial condition, that will be determined by the final situation after a pulse. This expression has been previously obtained in a different context [13]. For large times $G^{(0)}(y, t)$ approaches the limit

$$
G^{(0)}(y, \infty)=G_{0}(-1),
$$

and, consequently,

$$
\lim _{t \rightarrow \infty} D_{0}^{(0)}(t)=G_{0}(-1), \quad \lim _{t \rightarrow \infty} D_{r}^{(0)}(t)=0
$$

$r \geqslant 1$. The last result reflects the property that for $\epsilon=0$ all the holes are isolated in the long time limit, i.e., they are always between two particles. Therefore, the probability of finding two consecutive sites with $m_{i}=1$ is null. This is a general property that does not depend on the initial conditions. Of course, the asymptotic value of $D_{0}^{(0)}=\left\langle m_{i}\right\rangle^{(0)}$ is determined by the initial state of the system, being smaller than its initial value. It must be noticed that the hierarchy (17) admits as a stationary solution any constant value for $D_{0}$ as long as $D_{r, s t}=0$ for $r \geqslant 1$.

An interesting particular case is when the system is at equilibrium with a given value of $\epsilon>0$ before being suddenly changed to $\epsilon=0$. In terms of the temperature introduced in Sec. II this is equivalent to a quench of the system to $T=0^{+}$. The initial condition for this process is now [see Eq. (8)],

$$
D_{r}(0)=\epsilon^{r+1}
$$

Then

$$
G_{0}(y)=\epsilon e^{\epsilon y}
$$

and Eq. (22) yields

$$
D_{0}^{(0)}(\infty)=\epsilon e^{-\epsilon}
$$

For a purely random initial distribution $(\epsilon=1 / 2)$ it is $D_{0}^{(0)}(\infty) \simeq 0.3033$, i.e., less than one third of the sites are empty in the final metastable state, characterized by a "frozen" configuration.

In the above discussion there was no need for considering the time evolution of the distributions $C_{r, s}(t)$ defined in Eq. (16). Nevertheless, it is evident that in the limit $t \rightarrow \infty, C_{0,0}^{(0)}$ approaches a constant value fixed by the initial conditions of the relaxation process, while $C_{r, s}^{(0)}(t) \rightarrow 0$ for $r>0$ or $s>0$, since the last ones involve adjacent sites.

Next we analyze the evolution of the system with $\epsilon>0$ but for a time interval $t_{0} \ll 1$. This corresponds to the pulse preceding, and also following, each of the free relaxations without desorption. Therefore, the initial conditions we will be interested in correspond to a final state obtained after a long time relaxation with $\epsilon=0$,

$$
\begin{gathered}
D_{0}(0)=m_{0}, \quad D_{r}(0)=0 \text { for } r \geqslant 1, \\
C_{0,0}(0)=c_{0}, \quad C_{r, s}(0)=0 \quad \text { for } r \geqslant 1 \quad \text { or } s \geqslant 1 .
\end{gathered}
$$

For times $t \leqslant t_{0} \ll 1$ we approximate by means of a first-order Taylor expansion using Eqs. (13)-(15),

$$
D_{0}(t) \simeq m_{0}+\epsilon m_{0} t,
$$

and similarly

$$
\begin{gathered}
D_{1}(t) \simeq \epsilon\left(m_{0}+c_{0}\right) t, \\
D_{2}(t) \simeq \epsilon c_{0} t,
\end{gathered}
$$

while distributions $D_{r}(t)$ with $r \geqslant 3$ are at least of order $t^{2}$. Now, we can define the generating function corresponding to the pulse in a similar way as it was done for $\epsilon=0$ in Eq. (18). For this short time limit it is 


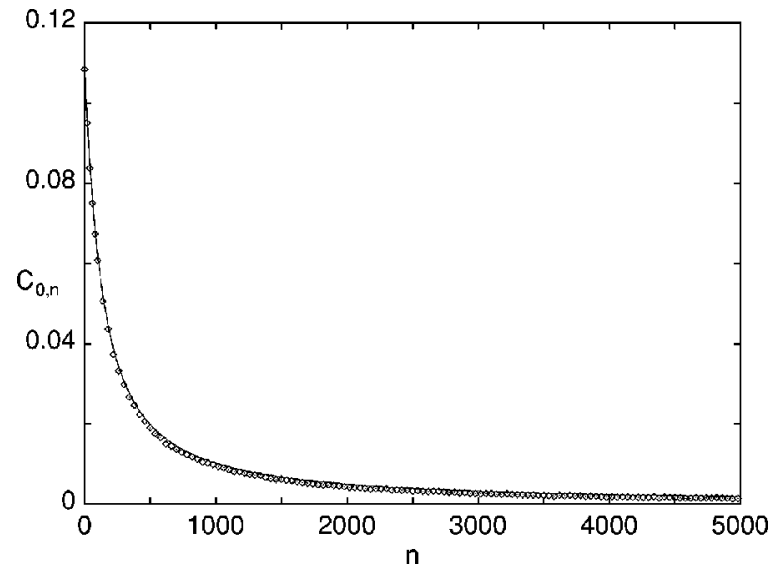

FIG. 1. Plot of both $c_{0, n}$ (solid line) and $2\left(\rho_{n+1}-\rho_{n}\right) / \epsilon t_{0}$ (diamonds) as functions of the number of taps $n$, for $\epsilon=0.5$ and $t_{0}$ $=0.02$.

$$
\begin{aligned}
G(y, t)= & \sum_{r=0}^{\infty} \frac{y^{r}}{r !} D_{r}(t)=m_{0}+t \epsilon m_{0}+t \epsilon\left(m_{0}+c_{0}\right) y \\
& +\frac{1}{2} t \epsilon c_{0} y^{2}+O\left(t^{2}\right) .
\end{aligned}
$$

\section{TAPPING PROCESSES}

In this section we will use the previous results to investigate the dependence of the density on the number of taps. Let us consider the free relaxation with $\epsilon=0$ after the $n$ +1 pulse. The initial condition for this process will be the final state reached during the pulse, i.e., using Eq. (31),

$$
\begin{aligned}
G_{n+1}^{(0)}(y, 0) \equiv & G_{0, n+1}(y)=m_{0, n}+t_{0} \epsilon m_{0, n}+t_{0} \epsilon\left(m_{0, n}+c_{0, n}\right) y \\
& +\frac{1}{2} t_{0} \epsilon c_{0, n} y^{2}+O\left(t_{0}^{2}\right),
\end{aligned}
$$

where $m_{0, n}$ and $c_{0, n}$ are the values of $\left\langle m_{i}\right\rangle$ and $\left\langle m_{i} m_{i+2}\right\rangle$ at the end of the relaxation following the $n$-th tap, respectively. The time evolution of the system during the relaxation is described by Eq. (20) and in the long time limit by Eq. (21), that particularized for the above initial condition yields

$$
G_{n+1}^{(0)}(y, \infty) \simeq m_{0, n}-\epsilon c_{0, n} \frac{t_{0}}{2}
$$

and, therefore,

$$
m_{0, n+1} \simeq m_{0, n}-\frac{1}{2} \epsilon t_{0} c_{0, n} .
$$

The above equation is expected to hold for small $t_{0}$ but arbitrary "amplitude" of the pulses $\epsilon$. Since $c_{0, n}$ is by definition positive it follows that the density of holes decreases and the system compacts monotonically as a function of the number of taps $n$. We stress that the density is measured at the end of each free relaxation as it is actually done in real experiments.

We have checked Eq. (34) by comparing it with the results obtained from Monte Carlo simulation of the Markov process defining the dynamics of the system. An example is given in Fig. 1 where we have plotted both $2\left(\rho_{n+1}\right.$ $\left.-\rho_{n}\right) / \epsilon t_{0}$ and $c_{0, n}$ as functions of the number of taps $n$. Here $\rho_{n}=1-m_{0, n}$ is the density of particles after the $n$th tap. In

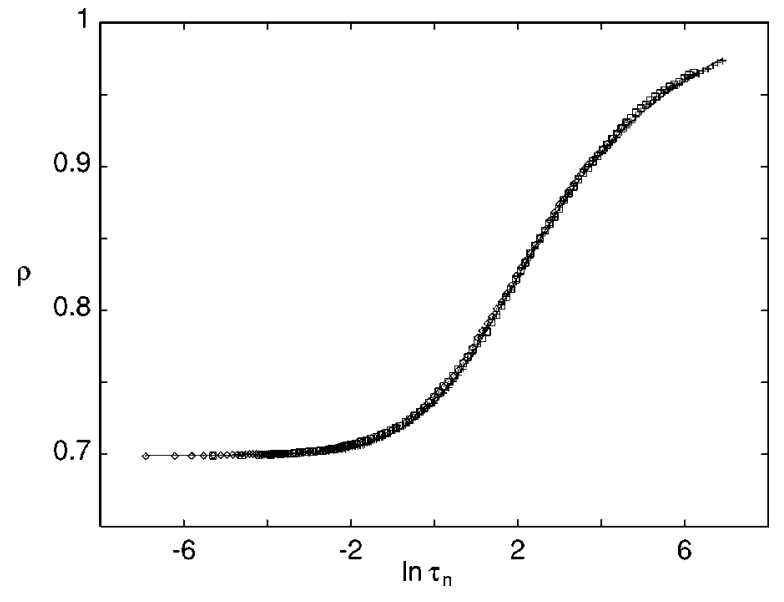

FIG. 2. Time evolution of the density of particles. Time is measured in the reduced scale defined in the text. In the three curves shown, the values of the parameters are $\epsilon=0.5$ in all of them and $t_{0}=2 \times 10^{-3}$ (diamonds), $t_{0}=0.01$ (squares), and $t_{0}=0.02$ (pluses).

fact, since $m_{0, n+1}-m_{0, n}$ is a rapidly fluctuating quantity, each of the points we have plotted corresponds to the average of those functions over 10 consecutive taps. The data shown have been obtained in a system of $10^{4}$ sites with $\epsilon=0.5$ and $t_{0}=0.02$, and have been averaged over $10^{3}$ runs. It is seen that the prediction of the theory is verified quite accurately. For the sake of clarity, we have restricted ourselves to 5 $\times 10^{3}$ taps, although the same behavior is observed until the system comes near the steady state discussed in the next section.

Equation (34) indicates that the compaction process depends on the product $\epsilon t_{0}$ and, in that sense, $\epsilon t_{0}$ plays in our model the same role as $\Gamma$ in real experiments. The latter is defined as the ratio of the peak acceleration of the tap to the gravitational acceleration [2]. Nevertheless, Eq. (34) suggests a stronger prediction, namely that the relevant time scale for the compaction process is $\tau_{n}=\epsilon t_{0} n$. Of course, this will be true only if the dependence of $c_{0, n}$ on $n$ also takes place through $\tau_{n}$, but it is easily seen that it is really so. The initial condition for each pulse is a trapped configuration that is metastable for $\epsilon=0$. That means that the derivatives with respect to time of all moments are proportional to $\epsilon$ at $t$ $=0$, and in the limit of short duration pulses the change in any moment in a pulse will be proportional to $\epsilon t_{0}$. This proportionality is clearly kept by the free relaxation with $\epsilon$ $=0$ that does not introduce any new time scale in the problem. It is worth mentioning that the same results, i.e., Eq. (34), hold in the limit $\epsilon \rightarrow 0$, with $\epsilon t_{0} \ll 1$.

In Fig. 2 the relaxation of the particle density is shown as a function of the scaled time $\tau_{n}$ for different values of $\epsilon$ and $t_{0}$. In all cases $t_{0} \ll 1$ as required by the theory we have developed. The system has $10^{4}$ sites. The initial state for all the compaction experiments was the same, namely the fully random distribution. As predicted, all points lie onto a single curve. Moreover, this scaled curve is well described by the four-parameter heuristic law

$$
\rho_{n}=\rho_{\infty}-\frac{\delta \rho_{\infty}}{1+B \ln \left(1+\frac{\tau_{n}}{\tau_{c}}\right)},
$$


with values of the parameters $\rho_{\infty}=1.10, \delta \rho_{\infty}=0.40, B$ $=0.39$, and $\tau_{c}=3.37$. We do not observe any dependence of these constants on the values of $\epsilon$ or $t_{0}$, of course always in the limit $t_{0} \ll 1$. The solid line in Fig. 2 is the fit to Eq. (35). We have tried to derive analytically a logarithmic law similar to this empiric result, but we have not succeeded. It is not clear yet whether it is just a convenient fitting expression with four parameters or it has a more fundamental meaning, for instance associated to some peculiar dynamical events which are dominant in the relaxation of the density. In this context, it is important to realize that the law fails to describe the asymptotic behavior in the limit of a large number of taps and the steady value of the density that is eventually reached. In fact, the value of $\rho_{\infty}$ reported above is clearly unphysical since it is larger than one. We believe that this is a general limitation of the law (35) and it is not restricted to the present model. We can substitute in Eq. (35)

$$
\frac{\tau_{n}}{\tau_{c}}=\frac{n}{n_{c}}
$$

with $n_{c}=\tau_{c} / \epsilon t_{0}$. In this way the standard inverse logarithmic law with time measured in number of taps is recovered [2]. But now we have an explicit dependence of $n_{c}$ on $\epsilon t_{0}$. A similar result was found numerically in Ref. [6] for a twodimensional model with geometrical frustration. Here the dependence appears as a consequence of the relevant scale defining the time evolution of the system. This scale has been identified by using analytical methods. The value $n_{c}$ can be understood as the minimum number of taps needed to observe a significant compaction process. For $n \ll n_{c}$ the density remains practically with its initial value.

\section{STEADY STATES}

Another point we have investigated, prompted, and stimulated by the results found in previous works by different authors, is the possible existence of a long time steady state density, determined by the tapping process (i.e., the amplitude and duration of the pulses in the present model) but independent of the initial conditions [5]. Then, we have carried out a series of computer experiments corresponding to the same values of $t_{0}$ and $\epsilon$ but to different initial conditions and, in particular, to different values of the initial density.

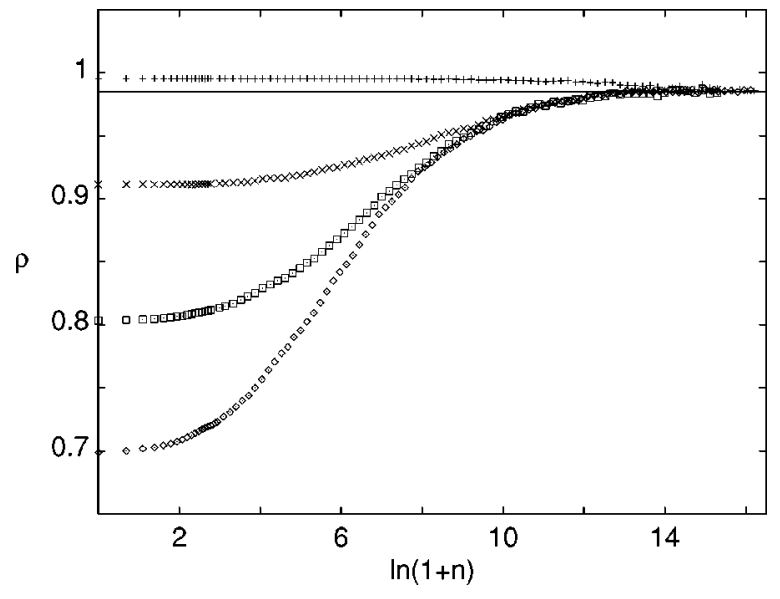

FIG. 3. Evolution of the density as a function of the number of taps, for four different values of the initial densities. The parameters characterizing the tapping process are $\epsilon=0.5$ and $t_{0}=0.06$ in all cases.

The states we have chosen are equilibrium states, corresponding to initial densities of particles $0.5,0.75,0.9$, and 0.995. The results are presented in Fig. 3. All densities tend in the long time limit to the same steady value. The data in the figure have been obtained in a system with $\epsilon t_{0}=0.03$, but the same qualitative behavior has been found in all the studied cases. One important point to remark is that it is possible to start from a density higher than the asymptotic one and then the density decreases as the number of tapings increases. Once again, this behavior is analogous to what is observed in real experiments. Average densities above the so-called random close packing limit, which is much smaller than the crystalline value, are not obtained even after extensive vibratory settling.

If we look for steady solutions of Eq. (34), the only consequence we can reach is that such state requires $c_{0}$ to be much smaller than $m_{0}$. A more specific statement can be obtained by considering the next order in the expansion of powers of $t_{0}$. Besides, we have simplified the calculations by considering a pair approximation for all the correlation functions. More specifically, we neglected all correlations involving more than two sites and approximated in Eqs. (13) and (14)

$$
\begin{gathered}
D_{2}(t) \equiv\left\langle m_{i} m_{i+1} m_{i+2}\right\rangle_{t} \simeq \frac{D_{1}^{2}(t)}{D_{0}(t)} \\
C_{0,0}(t) \equiv\left\langle m_{i} m_{i+2}\right\rangle_{t}=\left\langle m_{i} m_{i+1} m_{i+2}\right\rangle_{t}+\left\langle m_{i}\left(1-m_{i+1}\right) m_{i+2}\right\rangle_{t} \simeq \frac{D_{1}^{2}(t)}{D_{0}(t)}+\frac{\left[D_{0}(t)-D_{1}(t)\right]^{2}}{1-D_{0}(t)} .
\end{gathered}
$$

The above approximations can be shown to be equivalent to the dynamical mean field of clusters introduced by Dickman [14]. When Eqs. (37) and (38) are substituted into Eqs. (13) and (14),the latter become a close pair of nonlinear first order differential equations, namely

$$
\frac{d D_{0}(t)}{d t}=-D_{1}(t)+\epsilon D_{0}(t)
$$




$$
\frac{d D_{1}(t)}{d t}=-D_{1}(t)-\frac{D_{1}^{2}(t)}{D_{0}(t)}+\epsilon D_{0}(t)+\epsilon\left\{\frac{D_{1}^{2}(t)}{D_{0}(t)}+\frac{\left[D_{0}(t)-D_{1}(t)\right]^{2}}{1-D_{0}(t)}\right\} .
$$

In the $n+1$ pulse we have to solve the above equations, for the time $t_{0} \ll 1$ that the vibration lasts. Then, a perturbative solution in powers of $t_{0}$, with the initial conditions given by

$$
D_{0}(t=0)=m_{0, n}, \quad D_{1}(t=0)=0,
$$

is easily obtained,

$$
\begin{gathered}
D_{0}\left(t_{0}\right)=m_{0, n}+\epsilon t_{0} m_{0, n}+\frac{t_{0}^{2}}{2}\left(-\epsilon m_{0, n}+\epsilon^{2} m_{0, n}-\frac{\epsilon m_{0, n}^{2}}{1-m_{0, n}}\right)+O\left(t_{0}^{3}\right), \\
D_{1}\left(t_{0}\right)=t_{0}\left(\epsilon m_{0, n}+\frac{\epsilon m_{0, n}^{2}}{1-m_{0, n}}\right)+\frac{t_{0}^{2}}{2}\left[-\epsilon m_{0, n}+\epsilon^{2} m_{0, n}-\frac{\epsilon m_{0, n}^{2}}{1-m_{0, n}}-\frac{\epsilon^{2} m_{0, n}^{3}}{\left(1-m_{0, n}\right)^{2}}\right]+O\left(t_{0}^{3}\right) .
\end{gathered}
$$

Afterwards, the system evolves freely with $\epsilon=0$, and we measure the density of holes $m_{0, n+1}$ at the end of this relaxation. We have to solve Eqs. (39) and (40) with $\epsilon=0$. Writing them as a closed second order equation for the density of holes $D_{0}^{(0)}$, it is found that

$$
\frac{d}{d t}\left[\frac{d \ln D_{0}^{(0)}(t)}{d t}+\ln D_{0}^{(0)}(t)\right]=0
$$

and then

$$
\frac{d \ln D_{0}^{(0)}(t)}{d t}+\ln D_{0}^{(0)}(t)=-\frac{D_{1}\left(t_{0}\right)}{D_{0}\left(t_{0}\right)}+\ln D_{0}\left(t_{0}\right)
$$

since the initial conditions for the relaxation process are the final state of the pulse, given by Eqs. (42) and (43). The value of the density at the end of the relaxation is the long time limit solution of the above equation, i.e.,

$$
m_{0, n+1}=D_{0}^{(0)}(\infty)=D_{0}\left(t_{0}\right) e^{-D_{1}\left(t_{0}\right) / D_{0}\left(t_{0}\right)},
$$

and using Eqs. (42) and (43),

$$
\begin{aligned}
m_{0, n+1}= & m_{0, n}-\epsilon t_{0} \frac{m_{0, n}^{2}}{1-m_{0, n}} \\
& +\frac{1}{2}\left(\epsilon t_{0}\right)^{2} m_{0, n} \frac{1+m_{0, n}^{2}}{\left(1-m_{0, n}\right)^{2}}+O\left(t_{0}^{3} m_{0, n}\right) .
\end{aligned}
$$

As long as $m_{0, n}$ is much larger than $\epsilon t_{0}$ the third term on the right hand side is negligible as compared with the second one, and the density of holes decreases monotonically, i.e. the system is compacting. To the order or approximation considered in Eq. (47) a steady value of the density will be reached when

$$
m_{0, n+1}-m_{0, n}=O\left(t_{0}^{3} m_{0, n}\right)
$$

To get an expression for this steady density that we will denote by $m_{0}^{(s)}$, let us assume that it is of the order of $t_{0}^{\beta}$, $m_{0}^{(s)}=c t_{0}^{\beta}$. Therefore,

$$
\begin{aligned}
m_{0, n+1}-m_{0, n}= & -\epsilon t_{0}^{1+2 \beta} c^{2}+\frac{1}{2} \epsilon^{2} t_{0}^{2+\beta} c+O\left(t_{0}^{3+\beta}\right) \\
& +O\left(t_{0}^{1+3 \beta}\right)+O\left(t_{0}^{2+2 \beta}\right),
\end{aligned}
$$

and a simple dominant balance of the first and second terms on the right hand side of Eq. (47) yields $\beta=1$ and $c=\epsilon / 2$, i.e.,

$$
m_{0}^{(s)}=\frac{1}{2} \epsilon t_{0}
$$

We have discarded a solution $m^{(s)}=0$ that is always a trivial fix point for the evolution of the system, corresponding to all sites being occupied by particles. Let us notice that the above expression for $m_{0}^{(s)}$ is a steady solution of the evolution equations in the pair approximation up to and including order $t_{0}^{3}$. It is important to note that the steady value in the tapping process $m_{0}^{(s)}$ depends both on the pulse strength $\epsilon$ and its duration $t_{0}$. Therefore, $m_{0}^{(s)}$ does not coincide with the stationary value $\left\langle m_{i}\right\rangle_{s t}$ of Eq. (9), which would be the asymptotic value reached if the system was submitted to one tap with strength $\epsilon$ and infinite duration.

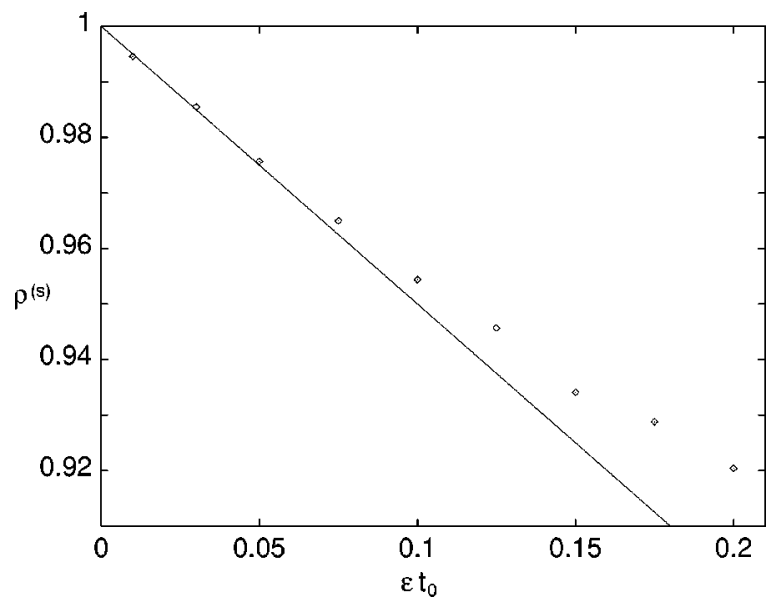

FIG. 4. Stationary value of the density of particles, $\rho^{(s)}=1$ $-m_{0}^{(s)}$, as a function of the parameter $\epsilon t_{0}$ characterizing the tapping process. The diamonds are numerical results, while the solid line is the analytical expression given by Eq. (50). 
In Fig. 3 we have indicated the prediction of Eq. (50). An excellent agreement is found with the steady value reached in the simulations. A comparison for several values of $\epsilon t_{0}$ is given in Fig. 4. Again the theory describes fairly well, both qualitatively and quantitatively, the results of the numerical simulations. The discrepancies increases as the value of $\epsilon t_{0}$ increases, as expected. This confirms that in the compaction experiment we are considering the steady correlations are determined mainly by the two nearest neighbor (pair) correlations. This is due to the fact that in the free relaxation processes the dynamics of the system is governed by the restriction that there cannot be a hole next to another one, but they are isolated, i.e., surrounded by particles.

\section{FINAL REMARKS}

In the framework of a simple one-dimensional lattice model with facilitated dynamics, we have studied the nonequilibrium evolution of a system submitted to a tapping process. Trying to mimic what is done in real experiments, the evolution of the system was modelled by a series of two alternating steps. In the first one, the system evolves perturbed by an external energy source, while in the second one it freely relaxes towards a metastable configuration. The model has been shown to share many of the characteristic features of granular materials under tapping. In particular, the evolution of the density can be accurately described by means of an inverse logarithmic law with the tapping number.

The model introduced in this paper has a mathematical structure very similar to the "parking" model of E. BenNaim and coworkers [8]. In both models the elementary dynamical events are the adsorption and desorption of particles on a line, with some simple geometrical restrictions. In both models an inverse logarithmic behavior has been found, and in the case of Ref. [8] it has been seen analytically. Nevertheless, there are physically relevant differences between both models. In the parking model the duration and strength of the taps as well as the free relaxation between them do not appear in the formulation. The transition rates of the master equation are considered as constant along the tapping process. In this context, it could be said that Ben-Naim's model provide an effective description of tapping processes, while the one here can in principle be used to describe more general processes.

In our model, compaction is due to the decrease of the number of holes as the system is being tapped. A qualitative picture of this very slow relaxation follows from the existence of "entropic barriers"' in the system. As the number of holes lowers, the number of states allowing the system to relax become very small as compared with the total number of available states. Therefore, it is very difficult for the system to find the way to these bottlenecks in configuration space, being most of the time trapped exploring metastable configurations with almost the same density. This picture supports a strong relationship between structural glassy relaxation and compaction [15]. In both cases there is a fast increase of the relaxation time of the system, becoming very large on the time scale of the experiment.

Due to the simplicity of the model we have been able to obtain some detailed analytical results. The process is characterized by the product $\epsilon t_{0}$, that identifies the relevant time scale for densification, at least in the limit of very short taps. Over this scale the time evolution of the system is described by a universal law, which is independent of the particular values of the parameters defining the system. This prediction has been compared with the numerical solutions obtained by Monte Carlo simulation and a very good agreement has been found.

A main result of this paper is an analytical expression for the asymptotic density obtained in a tapping process. Quite surprisingly, in the limit considered there is a very simple proportionality relation between this density and both the duration of the taps and their strength, the latter being measured by the relative probability of a desorption event during a tap. Also this theoretical prediction has been confirmed by the numerical solution.

\section{ACKNOWLEDGMENT}

This research was partially supported by the Dirección General de Investigación Científica y Técnica (Spain) through Grant No. PB96-0534.
[1] G. C. Barker and A. Mehta, Phys. Rev. A 45, 3435 (1992); Phys. Rev. E 47, 184 (1993).

[2] J. B. Knight, C. G. Frandich, C. N. Lau, H. M. Jaeger, and S. R. Nagel, Phys. Rev. E 51, 3957 (1995).

[3] E. R. Nowak, J. B. Knight, E. Ben-Naim, H. M. Jaeger, and S. R. Nagel, Phys. Rev. E 57, 1971 (1998).

[4] C. D. Hong, S. Yue, J. K. Rudra, M. Y. Choi, and Y. W. Kim, Phys. Rev. E 50, 4123 (1994).

[5] E. R. Nowak, J. B. Knight, M. Povinelli, H. M. Jaeger, and S. R. Nagel, Powder Technol. 94, 79 (1997).

[6] E. Caglioti, V. Loreto, H. J. Herrmann, and M. Nicodemi, Phys. Rev. Lett. 79, 1575 (1997).

[7] M. Nicodemi, A. Coniglio, and H. J. Herrmann, Phys. Rev. E 55, 3962 (1997).
[8] E. Ben-Naim, J. B. Knight, E. R. Nowak, H. M. Jaeger, and S. R. Nagel, Physica D 123, 380 (1998).

[9] H. M. Jaeger (unpublished).

[10] N. G. van Kampen, Stochastic Processes in Physics and Chemistry (North-Holland, Amsterdam, 1992).

[11] G. H. Fredrickson and H. C. Andersen, Phys. Rev. Lett. 53, 1244 (1984); J. Chem. Phys. 83, 5822 (1985).

[12] S. F. Edwards and R. B. S. Oakeshott, Physica A 157, 1080 (1989); S. F. Edwards and C. C. Mounfield, ibid. 210, 279 (1994).

[13] E. Follana and F. Ritort, Phys. Rev. B 54, 930 (1996).

[14] R. Dickman, Phys. Rev. A 34, 4286 (1986); Phys. Lett. A 122, 463 (1987).

[15] L. P. Kadanoff, Rev. Mod. Phys. 71, 435 (1999). 